Volume 1

Issue 1 Transformative Possibilities:

Transcending Interlocking Boundaries

\title{
Performance and Application of an Inexpensive Method for Measurement of Nitrogen Dioxide
}

Jeremy Parra

Portland State University

Follow this and additional works at: https://pdxscholar.library.pdx.edu/mcnair Let us know how access to this document benefits you.

\section{Recommended Citation}

Parra, Jeremy (2004) "Performance and Application of an Inexpensive Method for Measurement of Nitrogen Dioxide," PSU McNair Scholars Online Journal: Vol. 1: Iss. 1, Article 10.

https://doi.org/10.15760/mcnair.2005.278

This open access Article is distributed under the terms of the Creative Commons Attribution-NonCommercialShareAlike 4.0 International License (CC BY-NC-SA 4.0). All documents in PDXScholar should meet accessibility standards. If we can make this document more accessible to you, contact our team. 
Portland State University McNair Research Journal 2004-2005

Performance and Application of an Inexpensive Method for Measurement of Nitrogen Dioxide by

Jeremy Parra

Faculty Mentor:

Linda George

Citation: Parra, Jeremy. Performance and Application of an Inexpensive Method for Measurement of Nitrogen Dioxide. Portland State University McNair Scholars Online Journal, Vol. 1, 2004-2005: 278-286. 


\title{
Performance and Application of an Inexpensive Method for Measurement of Nitrogen Dioxide
}

\author{
Jeremy Para \\ Faculty Mentor: Linda George
}

\begin{abstract}
$\underline{\text { Abstract }}$
To make air quality monitoring accessible to a broad range of people (i.e. consumers, educational groups, and environmental activist) a low-cost method for sampling nitrogen dioxide (NO2) was modeled after the Palmes and Gunnison diffusion samplers. This article describes the results of using cost-effective passive diffusion tubes for measuring NO2 in outdoor environments. Samplers are compared against a collocated chemiluminescence (active) monitor in a gas exposure chamber for concentration of NO2 between 10 and $200 \mathrm{ppb} / \mathrm{v}$ (parts per billion per volume). In these calibration experiments diffusion samplers had strong correlation to the active monitor, $\mathrm{R} 2=0.94$ for a one week sample interval, and a R2 $=0.91$ for a two week sample interval. A four week study was conducted with a collocated active monitor and a total of 42 samplers. The samplers were divided into 6 groups and exposed in one week and two week intervals under environmental conditions: temperature $18-31^{\circ} \mathrm{C}$, relative humidity $27-57 \%$, wind speed $0.4-3 \mathrm{~m} / \mathrm{s}$, and NO2 concentration $2-22 \mathrm{ppb} / \mathrm{v}$. The precision, calculated as the relative standard deviation, for the one week sample interval was found to be $8.2 \%$, and for the two week sample interval $20.2 \%$. Three cleaning methods of exposed samplers are compared in order to identify bias in reusing components. Using the best cleaning method the mean NO2 absorbance of a dry sampler is $1.3 \pm$ $0.4 \mathrm{ppb} / \mathrm{v}$ as opposed to a zero absorbance for unused samplers.
\end{abstract}

\section{Introduction}

With more people becoming aware of the negative impact that transportation and industry pollutants are having on their health1, the need for low-cost air sampling devices has become predominant. Nitrogen oxides (NOx) are key urban pollutants linked to respiratory illnesses 2 . NOx are formed when fuel is burned at high temperatures, as in combustion exhaust. Mostly nitrogen oxide (NO) is produced in the combustion process but it is readily oxidized in the atmosphere to produce $\mathrm{NO} 2(\mathrm{NO}+\mathrm{O} 2=>\mathrm{NO} 2)$. Because sources of NOx are primarily anthropogenic, a community's exposure is highly influenced by its proximity to roadways and industry plants. Also, because NO2 is a bi-product of combustion, it acts as a tracer for other noxious pollutants such as hydrocarbons, carbon monoxide, and surface level ozone 3.

Traditionally, the concentration of $\mathrm{NO} 2$ is actively sampled using instruments such as the chemiluminescence analyzer 2 which can monitor one point in space continuously. Developing networks of these instruments, which would show the distribution of NO2, is hampered by their high cost 4. Passive diffusion samplers (sampler) can be utilized as a relatively inexpensive method ( $\sim$ \$1.50 per sampler) for measuring ambient NO2. Because of their low cost it is relatively 
affordable to put samplers at multiple sites within a study region. Although the measurement represents a time average (in the scale of weeks) we can continue a study for many months at very low cost. A great advantage of the passive diffusion tube is its ease of use; because of this it is feasible to get multiple groups (high school students, churches, community action groups) involved in air monitoring campaigns for several months at a time. For these reasons there is a great deal of incentive to incorporate diffusion tubes into an air monitoring protocol.

The use of passive diffusion tubes for measuring NO2 was first introduced by Palmes and Gunnison 5. Initially they were used for workplace monitoring, but have been adapted for air quality management ${ }^{6}-9$. Diffusion is a property of gases by which a gas will flow from a place of higher concentration to a place of lower concentration. If a suitable absorbing agent is used, the concentration of a gas can be kept theoretically zero at a point. Thus gas will always flow to that point. With this property in mind, we can constrict the flow of a sample to a tube, having one end open to the gas sample and the other end closed off with the suitable absorbing agent present at the closed end. The absorbing agent used for NO2 is triethanolamine (TEA), when NO2 in the atmosphere diffuses into the sampler it reacts with the TEA and is converted to nitrite. The nitrite remains in the TEA and more NO2 diffuses into the sampler. The rate at which NO2 diffuses into the tube is found experimentally to be $0.154 \mathrm{~cm} 2 \mathrm{~s}-1$. When the sampling interval is concluded, the mass of nitrite (Q) absorbed in the TEA is determined using UV-spectroscopy; Q is proportional to the concentration of NO2 in the sampled and the length of exposure.

There still remain many questions as to the confidence that can be placed in this method 5,10-14. In a study by Plaisance et al. (2002) several biases resulting from meteorological parameters are identified, specifically overestimation caused by wind turbulence. In their work, a protective shelter (originally proposed by Hangartner et al. 1987) is suggested as well as a corrective formula arrived at by experiments at various wind speeds. Other works point out potential overestimation from "within the tube" chemistry 13.

It was found in an inter-laboratory comparison that differences in sampler preparation are a main source of inaccuracy14. Therefore, the sampler preparation and analysis procedure are outlined. In our field studies a question arose concerning the reuse of samplers, thus three cleaning methods of sampler components are compared.

\section{The sampler}

\subsection{Sampler design}

Samplers (figure 1) are made from industrial grade acrylic tubing with dimensions: length $=7 \mathrm{~cm}$ and internal diameter $=0.95 \mathrm{~cm}$ (Multi-Craft Plastics, Inc. (503) 288-5131). A triethanolamine (TEA, 98\%) absorbing solution of 20\% TEA in aq. is prepared by combining $20 \mathrm{ml}$ of TEA, $80 \mathrm{ml}$ of distilled water, and $160 \mu \mathrm{L}$ wetting solution (1 gram of Brij-35 dissolved in $9 \mathrm{ml}$ distilled water). Two caps (also of Industrial Acrylic) are secured to the ends of the tube. One cap is pressed down 
tightly onto the tube, this cap houses two TEA-coated stainless steel wire mesh screens (40 x 40 mesh); it is here that NO2 is converted to nitrite. A second cap is loosely placed on the open end of the tube before and after exposure.

figure 1: Sampler Construction

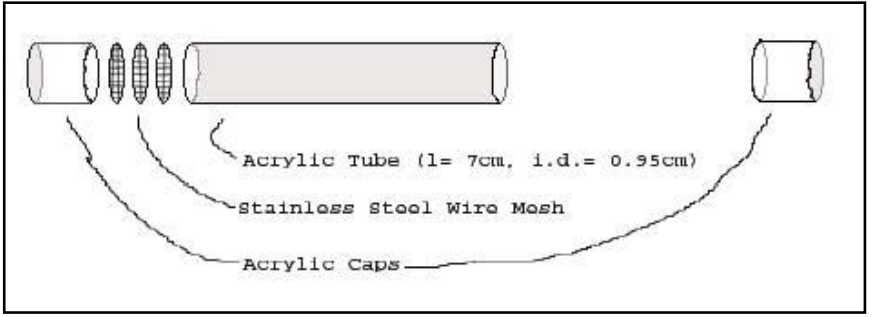

The samplers are stored in a refrigerator (at $9^{\circ} \mathrm{C}$ ) until deployment. When ready to use, the sampler is secured at least $4 \mathrm{~cm}$ from any surface by steel wire to a convenient object (e.g. telephone pole). After siting the sampler the cap from the end without screens is removed and washed. The sampler is hung so that the open mouth of the tube is facing downward. After the sampling interval is concluded the previously removed end cap is loosely replaced.

\subsection{Theory of Diffusion}

The theoretical background for the passive diffusion sampler was worked out by Palmes et al. 1976; specifically an estimate of the diffusion coefficient for NO2 in air is given. The equation describing the rate of diffusion is:

$\left[\mathrm{NO}_{2}\right]=\frac{Q \cdot z}{D_{\mathrm{NO}_{2}} \cdot A \cdot t}$, where

$\mathrm{Q}=$ mass of nitrite $(\mu \mathrm{g})$,

$\mathrm{z}=$ diffusion length $(\mathrm{cm})$,

$\mathrm{D}_{\mathrm{NO}_{2}}=$ diffusion coefficient for NO2 in air (cm2 $\left.\cdot \mathrm{s}-1\right)$,

$\mathrm{A}=$ internal cross-sectional area of tube $(\mathrm{cm} 2)$ and

$\mathrm{t}=$ collection time $(\mathrm{s})$.

The best estimate for the diffusion coefficient was found 5 to be $0.154 \mathrm{~cm} 2 \cdot \mathrm{s}^{-} 1$, supplying the diffusion coefficient, length and i.d. of the sampler the total equation is, $\mathrm{DO}_{2}$

$\left[\mathrm{NO}_{2}\right] p p b=\frac{Q(M)}{t} \cdot 2.87 \times 10^{12}$

The free variables are $\mathrm{Q}$ mass of nitrite and the time of exposure in seconds.

\subsection{Determining concentration of nitrite by UV-spectroscopy}


The mass of nitrite is determined by UV- spectroscopy using a Bausch and Lomb UV- spec 20. The samplers off the field are stored in a refrigerator until analysis. The samplers are placed in a rack with the screened end down. The loose end caps are removed. Using a volumetric pipette $1.6 \mathrm{ml}$ of analytic solution and $1.4 \mathrm{ml}$ of distilled water are measured directly into the diffusion tube. The analytic solution is made up off seven parts sulfanilamide solution to one part NEDA solution (see below for preparation of solutions). When this solution reacts with the nitrite "trapped" in the TEA it begins to color proportionally to the amount of nitrite present. A spectrometer (measuring at 540 $\mathrm{nm})$ is used to measure the absorbance of the sample.

To determine the coefficient of proportionality between the dye absorption and quantity of nitrite a calibration curve is created. Five solutions with varying concentration of nitrite are prepared, called standard solution 1-5. In five marked test tubes, one test tube for each concentration, $1.4 \mathrm{ml}$ of the corresponding standard solution is combined with $1.6 \mathrm{ml}$ of analysis solution. The concentration is

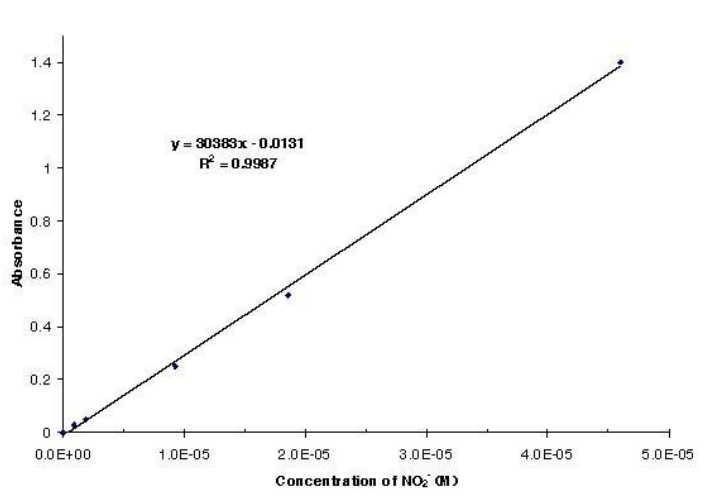
plotted against the UV absorbance (figure 2). This equation is unique to each analysis solution. For a calibration curve to be accepted it must have a R2 > 0.999 and an intercept $<|0.1|$. The sulfanilamide solution is prepared by weighing 5 grams of sulfanilamide dissolved in $15 \mathrm{ml}$ of phosphoric acid. The solution is diluted to $250 \mathrm{ml}$ with distilled water. The NEDA solution is prepared by diluting 0.35 grams of NEDA to $250 \mathrm{ml}$ with distilled water.

figure 2: Sample Calibration Curve

\section{Experimental}

\subsection{Determining offset absorbance}

\subsubsection{Unexposed samplers used to determine leak absorbance}

There are a few instances when a sampler is exposed to ambient air which does not represent the in situ NO2 concentration; as is the case when assembling and preparing the sampler for absorbance measurements. We would like to make a track record of these renegade measurements in order to subtract them from the final result. This is managed by including an extra sampler that remains unexposed (blank sampler). The blank sampler is prepared in the same manner as the others, but the end cap is not removed until all the samplers are brought in for analysis. One blank sampler was included in each of the six groups put in the field. The mean concentration for these was $2 \pm 2$ $\mathrm{ppb} / \mathrm{v}$ [NO2] (parts per billion per volume). 


\subsubsection{Comparison of Cleaning Methods}

Part of maintaining low cost is reusing samplers. Thus, an effective cleaning method is needed to remove the residual TEA solution, which has absorbed nitrite in it, from the sampler components (i.e. caps, tube, and wire mesh screens).

Twenty-eight samplers used in a preliminary field study were employed in this experiment. The acrylic tubes and end caps were washed with an aqueous solution of 3\% triton X-100 (cleaning solvent) in an ultrasonic bath for one hour. The components were then rinsed with distilled water and set to air dry.

The wire mesh screens (where most of the TEA is deposited) were divided into three groups and each group was washed in a different manner. In the first method screens were soaked in distilled water and dish soap $(n=9)$. For the second method, screens were soaked in phosphoric acid $(n=9)$. In both methods the screens were placed in a beaker and frequently agitated for 20 minutes. In the third method the screens were bathed in an ultra sonic bath with a 3\% triton X-100 in aq. solution for half an hour $(n=10)$. After each method the screens were thoroughly rinsed in distilled water and let to air dry.

The samplers were then exposed for a period of three days at an average concentration of $10 \mathrm{ppb} / \mathrm{v}$ [NO2] in the gas exposure chamber. TEA absorbing solution was not added, so that NO2 absorbance was do to residual TEA from previous use.

\subsubsection{Results of Cleaning and Unexposed samplers}

The screens cleaned with dish soap had the lowest mean absorbance $1.3 \pm 0.4 \mathrm{ppb} / \mathrm{v}$ [NO2] (standard error for a 95\% confidence interval). The mean absorbance for the phosphoric acid methods were $2 \pm 1 \mathrm{ppb} / \mathrm{v}$ [NO2], and for Triton $\mathrm{x}-1002 \pm 0.7 \mathrm{ppb} / \mathrm{v}$ [NO2]. A comparison of the sample means at a $90 \%$ confidence level resulted in a difference in measurements between the soap/water method versus the phosphoric and triton X-100 methods. But, a difference could not be established between the phosphoric and triton X-100 methods at the $90 \%$ confidence level. A comparison of mean measurements of the cleaned samplers and the unexposed samplers also did not show any significant difference.

Thus, we can conclude that after the tubes have been used they may be washed with soap and water. Also, an unexposed sampler should be included in order to subtract the absorbance of this sampler from the actual in situ measurements. 


\subsection{Laboratory Calibration}

\subsubsection{The setup}

To determine the accuracy and precision of samplers an exposure chamber was constructed in the lab (figure 3). The chamber is a cylindrical Teflon bag with a $9 \mathrm{~L}$ volume. The desired amount of NO2 is generated by a multi-gas calibrator (Dasibi, 5008) and pumped into the bag camber at 3 liters

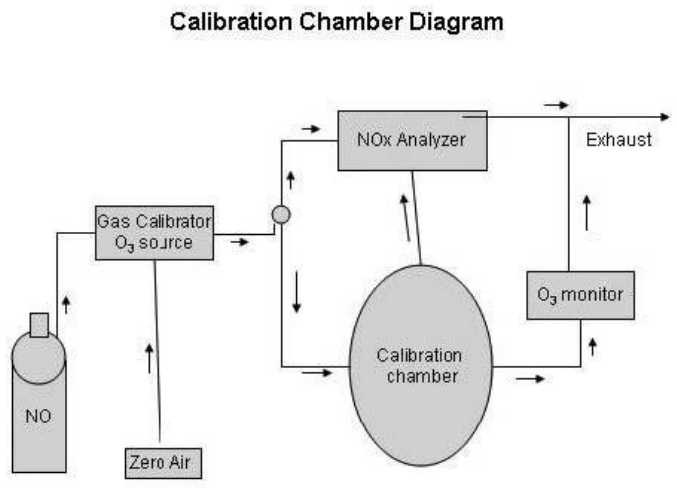
per minute through Teflon tubing with i.d. $=2 \mathrm{~mm}$. Ozone is generated by the multi-gas calibrator and mixed with $\mathrm{NO}$ in pure nitrogen mixture. NOx and Ozone concentrations were sampled continually using a chemiluminescence monitor (Thermo Environmental Inc., model 42c) and ozone monitor (Dasibi, 1003-AH), respectively. The contents were well mixed by a fan which hangs from the top of the chamber. Several measurements were made to insure that the wind speed within the bag never exceeded 1 $\mathrm{m} / \mathrm{s}$. The temperature was kept within a range of 18

$25^{\circ} \mathrm{C}$

figure 3: Calibration Chamber Diagram

\subsubsection{Results of laboratory calibration experiments}

Both the one-week and two-week intervals resulted in measurements well correlated to the chemiluminescence monitor. The one-week interval had a R2 $=0.91$ and the two-week, R2 $=0.94$. The offset for both sets is within the error range of the blank samplers (i.e. $2 \pm 2$ ). The slope for the one-week samplers is $0.9 \pm 0.1$. Whereas the slope for the two-week sample interval is 1.9 , it is not clear what the cause for this underestimation by half in the two-week data set. But, the laboratory measurements and in situ measurements seem to be consistent.

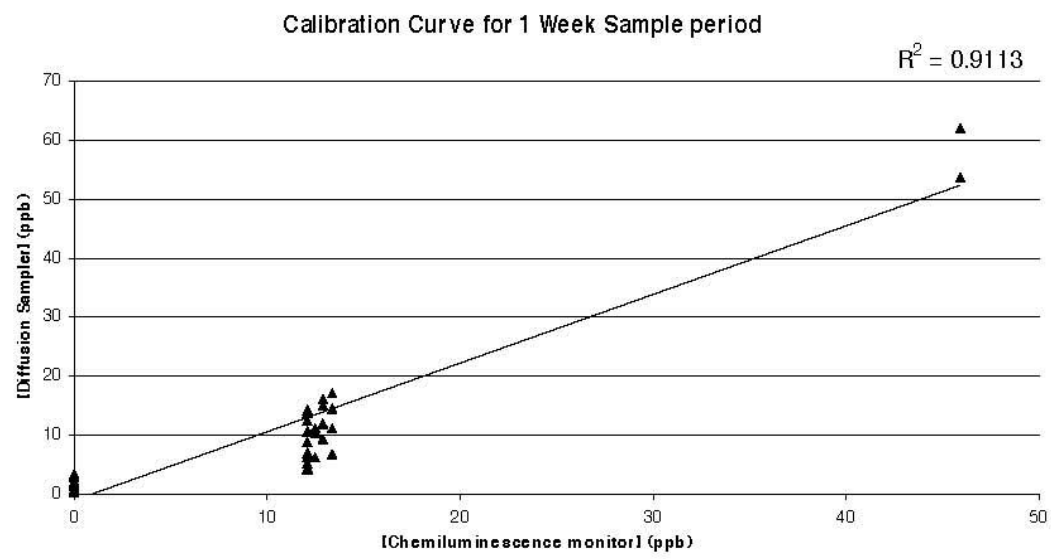

figure 4.a 


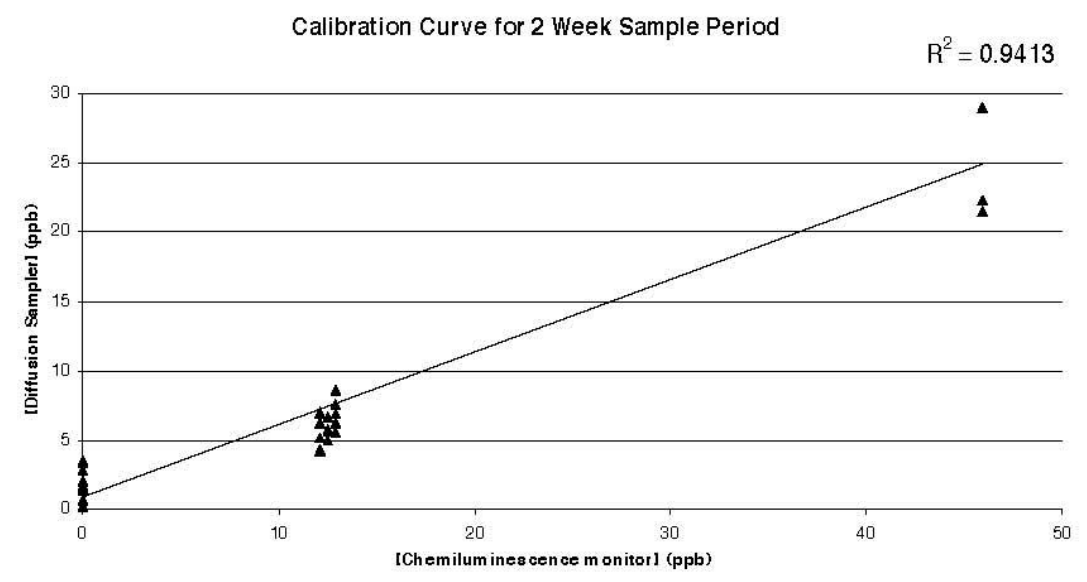

figure 4.b

\subsection{Collocated Active Sampling/ Diffusion Samplers}

\subsubsection{Data Collection}

A four-week outdoor study was made on the roof of the science building at P.S.U. The site was chosen because air quality and meteorological measurements are made continuously (ten-minute time scale) and are readily available through the Horizons' Network (www. nextgen.pdx.edu). The study was conducted in a four-week period under a range of environmental conditions: temperature $18-31^{\circ} \mathrm{C}$, relative humidity $27-57 \%$, wind speed $0.4-3 \mathrm{~m} / \mathrm{s}$, and NO2 $2-22 \mathrm{ppb} / \mathrm{v}$. The NO2 measurements were made from a thermo environmental chemiluminescence monitor. The diffusive samplers were divided into two groups and assigned randomly. Group A consisted of four sets of samplers exposed for one-week intervals in succession for the four-week period. Group B consisted of two sets of samplers exposed for two-week intervals. Each set was comprised of six samplers and one blank. The tubes were hung in close proximity to the intake of the active monitor.

\subsubsection{Results}

The accuracy of mean sampler (x) measurements is small, xmonitor - xsampler $<1.9$ [NO2] ppb/v. And in three cases the mean of the sampler measurement is exactly equal to the active monitor. The precision of the each set is measured as the standard error at the $95 \%$ confidence interval using the pooled sample standard deviations. The one-week sample interval has greater variance, \pm 2.4 [NO2] $\mathrm{ppb} / \mathrm{v}$, than the two-week interval, \pm 1 [NO2] $\mathrm{ppb} / \mathrm{v}$. While it cannot be concluded from these studies, other work has suggested a longer sample interval (i.e. two weeks) results in better precision. Our study seems to corroborate with this. The precision of the one-week sample interval measured as the relative standard deviation is $20.2 \%$, and the two-week sample interval is $8.2 \%$. Below are the results of the four-week outdoor study; the one-week sample interval in figure 5 and the two-week sample interval in figure 6. 
In Situ Comparison of Collocated Chemiluminescence Monitor and Diffusion samplers for one week sample interval

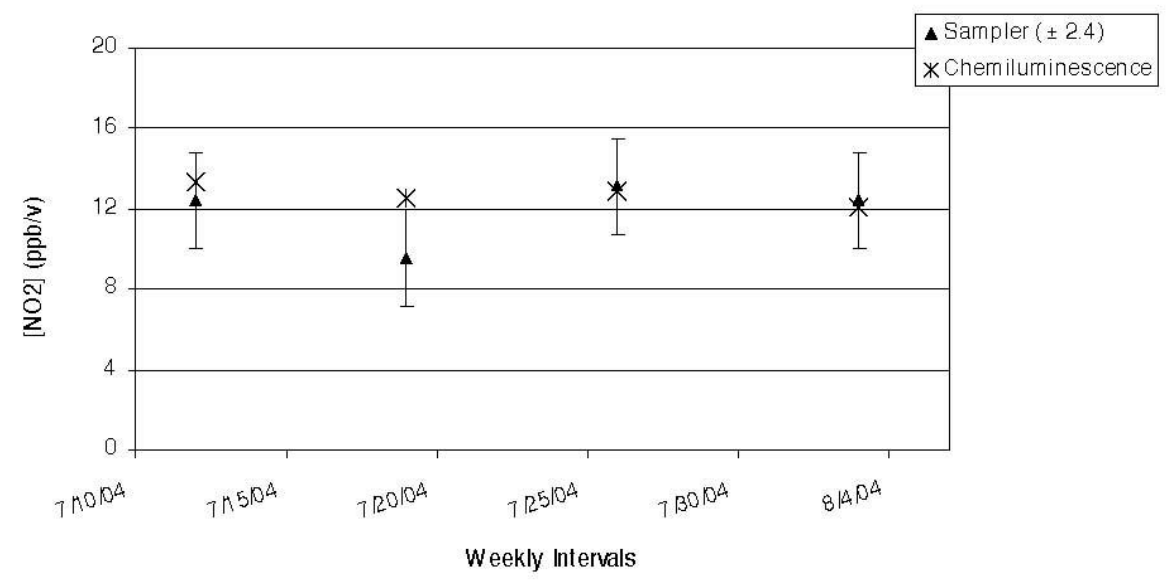

figure 5

In Situ Comparison of Collocated Chemilumin escen ce Monitor and Diffusion samplers for two week sample interval

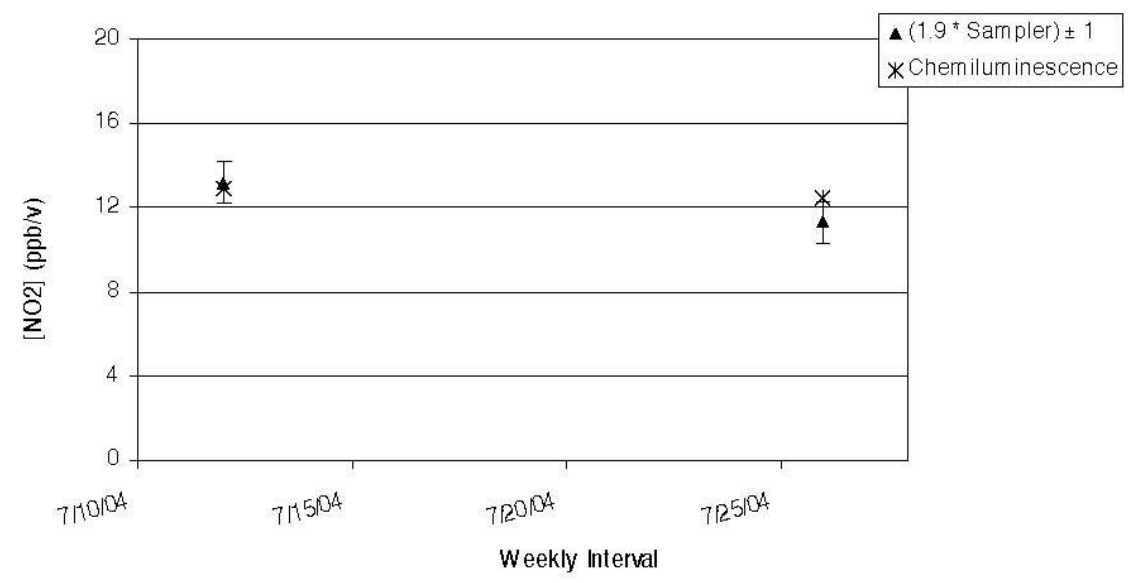

figure 6

\section{Discussion}

The results from three cleaning methods suggest that the diffusion sampler components should be washed in water and dish soap before reuse. Also, an extra diffusion sampler that is to remain unexposed should be included in all experiments utilizing the samplers. The concentration of NO2 determined by analyzing the extra sampler is the baseline measurement that should be subtracted from the rest of the data.

A four-week outdoor study suggests that diffusion tubes are accurate in their measurements of [NO2] compared to a chemiluminescence monitor. For better precision, a two-week sampling interval is preferred over a one-week sample interval. But this may not always be appropriate, in the case where a shorter time interval is needed, the precision of one week was still found to be good at $20.2 \%$. 
Laboratory calibrations of the diffusion samplers are still carried out at Portland State University. Also, multiple outdoor field studies in the Portland, Oregon metro area are currently in effect. It is the hope that this cheap method will be accepted as an accurate measure of nitrogen dioxide. 